\title{
Triglyceride-glucose Index Predicts 2-year Adverse Cardiovascular and Cerebrovascular Events in Patients With Type 2 Diabetes Mellitus and Non-st- segment Elevation Acute Coronary Syndrome Who Underwent Off-pump Coronary Artery Bypass Grafting
}

\section{Liang Chen}

Capital Medical University Affiliated Anzhen Hospital

Xiao-Hang Ding

Capital Medical University Affiliated Anzhen Hospital

Kang-Jun Fan

Capital Medical University Affiliated Anzhen Hospital

Ming-Xin Gao

Capital Medical University Affiliated Anzhen Hospital

Wen-Yuan Yu

Capital Medical University Affiliated Anzhen Hospital

Hong-Li Liu

Capital Medical University Affiliated Anzhen Hospital

Yang Yu ( $\sim$ heartyuyang@hotmail.com )

Capital Medical University Affiliated Anzhen Hospital https://orcid.org/0000-0002-8482-4626

\section{Research Article}

Keywords: MACCEs, TyG, OPCABG, NSTE-ACS, hypertension, triglyceride glucose

Posted Date: June 28th, 2021

DOI: https://doi.org/10.21203/rs.3.rs-644559/v1

License: (9) This work is licensed under a Creative Commons Attribution 4.0 International License. Read Full License 


\section{Abstract}

Background: Data on the relationship between the triglyceride glucose (TyG) index and prognosis after off-pump coronary artery bypass grafting (OPCABG) are limited. This retrospective observational study evaluated the association of the TyG index with prognosis in patients with type 2 diabetes mellitus (T2DM) and non-ST-segment elevation acute coronary syndrome (NSTE-ACS) who underwent OPCABG.

Methods: A total of 1534 patients with T2DM who underwent OPCABG for NSTE-ACS were enrolled in this study. Patients were divided into lower and higher TyG index groups according to the optimal cutoff value of the TyG index for predicting the primary endpoint. The primary outcomes included the occurrence of major adverse cardiovascular and cerebrovascular events (MACCEs), which were defined as all-cause death, nonfatal myocardial infarction, nonfatal stroke and symptomatic graft stenosis or occlusion. The TyG index was calculated using the following equation: TyG index $=\ln$ (fasting triglyceride level $[\mathrm{mg} / \mathrm{dL}] \times$ fasting glucose level $[\mathrm{mg} / \mathrm{dL}] / 2$ ).

Results: The overall incidence of MACCEs was $10.7 \%$ with a median follow-up of 23.1 months. The optimal TyG index cutoff for predicting MACCEs was 9.42 (sensitivity $49.4 \%$; specificity $71.2 \%$; area under the curve $0.631 ; P<0.001)$. The incidence of MACCEs increased with a 1-unit increase in the TyG index [HR 2.218, 95\% Cl (1.733-2.839), P < 0.001]. A TyG index greater than 9.42 was independently associated with a higher risk of MACCEs [HR 2.092, 95\% $\mathrm{Cl}$ (1.573-2.784), $\mathrm{P}<0.001$ ] and each component. Furthermore, the effect of TyG in increasing MACCEs was consistent across groups of age, sex, BMI, hypertension, LDL-C, oral hypoglycemic agents, LIMA, number of grafts and IABP groups, except in patients diagnosed with NSTEMI, treated with statins and prescribed insulin at discharge.

Conclusion: The TyG index predicts future MACCEs independently of known cardiovascular risk factors, suggesting that the TyG index may be a useful marker for prognosis in patients with T2DM and NSTEACS who underwent OPCABG.

\section{Introduction}

Coronary artery bypass grafting $(C A B G)$ remains the preferred treatment option for patients with severe multiple vessel coronary artery disease [1]. Although surgical techniques and medical treatment have been developed, the risk of recurrent adverse cardiovascular and cerebrovascular events (MACCEs) remains relatively high in patients who have had acute coronary syndrome (NSTE-ACS) [2].

The triglyceride-glucose (TyG) index, a product of triglycerides and fasting plasma glucose (FPG), is a good tool that correlates with surrogate and direct measures of insulin resistance (IR) $[3,4]$. Previous studies have reported that the TyG index is significantly associated with an increased risk of developing T2DM, hypertension and adverse cardiovascular events [5-8]. Moreover, several cross-sectional studies have reported a strong relation between the TyG index and atherosclerosis in different clinical conditions [9-11]. However, whether TyG may be associated with the adverse CV events of patients with T2DM and NSTE-ACS who were treated with OPCABG has not yet been assessed. Therefore, in the present study, we 
examined the relationship between the baseline TyG index and MACCE outcomes in 1534 Chinese patients with T2DM and NSTE-ACS who underwent OPCABG.

\section{Methods}

Study population and design

Data from the medical information recording system of Beijing Anzhen Hospital between February 2018 and December 2019 were analyzed in the present study. The exclusion criteria are listed in Fig. 1. In total, 1534 patients were retrospectively included in the final analysis. The present study was performed in accordance with the Helsinki Declaration of Human Rights and approved by the Institutional Review Board of Beijing Anzhen Hospital, Capital Medical University. Given the retrospective nature of this study, the requirement for informed consent was waived.

Data collection

Data including patient demographics, such as age, sex, BMI, current smoking status, current drinking status, heart rate, systolic blood pressure (SBP), diastolic pressure (DBP), laboratory results, surgical procedures and medical treatments, were obtained from the medical information recording system of Beijing Anzhen Hospital. Body mass index (BMI) was calculated using the following equation: $\mathrm{BMI}=$ weight $(\mathrm{kg}) /$ height $\left(\mathrm{m}^{2}\right)$. Blood samples were taken after overnight fasting $(>8 \mathrm{~h})$. Serum levels of fasting plasma glucose (FPG), glycated albumin (GA) and lipid profiles, including triglyceride (TG), total cholesterol (TC) and high-density lipoprotein cholesterol (HDL-C), were determined by standard laboratory techniques. The low-density lipoprotein cholesterol (LDL-C) level was computed with the Friedewald equation. The TyG index was computed using the following formula: TyG index $=\ln [$ fasting $T G(\mathrm{mg} / \mathrm{dL})$ $\times$ FPG $(\mathrm{mg} / \mathrm{dL}) / 2]$. The eGFR was calculated as previously described: eGFR $\left[\mathrm{mL} /\left(\min \times 1.73 \mathrm{~m}^{2}\right)\right]=186 \times$ serum creatinine $(\mathrm{mg} / \mathrm{dL})^{-1.154} \times$ age $^{-0.203}(\times 0.742$ if female) [12]. Left ventricular ejection fraction (LVEF) was evaluated by a two-dimensional modified Simpson's method using an ultrasonic cardiogram (Philips Company, Eindhoven, The Netherlands).

Coronary data and surgical details were recorded by two experienced cardiologists according to the surgical records. Single grafts, two grafts and multiple grafts were defined as using one, two and more than two grafts, respectively. We also grouped the type of graft as follows: single left internal mammary artery (single LIMA), single great saphenous vein grafts (single SVG), LIMA + SVG and all SVG.

\section{Definitions}

The criteria for diabetes were as follows: (1) previously diagnosed diabetes under treatment with antidiabetic medication (diet, oral agents and/or insulin); and (2) the typical symptoms of diabetes with a random blood glucose $\geq 11.1 \mathrm{mmol} / \mathrm{L}$ and/or FPG $\geq 7.0 \mathrm{mmol} / \mathrm{L}$ and/or 2-h blood glucose after oral glucose tolerance test (OGTT) $\geq 11.1 \mathrm{mmol} / \mathrm{L}$ [13]. NSTE-ACS was composed of non-ST-segment elevation myocardial infarction (NSTEMI) and unstable angina (UA), and the definitions of which were 
determined by appropriate guidelines [14]. NSTEMI was defined as having symptoms of ischemia and elevated cardiac troponin I (cTnl) without an elevation of the ST segment. UA was diagnosed as ischemic symptoms at rest and exacerbated or new-onset symptoms with transient ischemic ST-segment shifts without release of myocardial enzymes related to myocardial necrosis. Hypertension was defined as systolic blood pressure $\geq 140 \mathrm{mmHg}$ or diastolic blood pressure $\geq 90 \mathrm{mmHg}$, a previous diagnosis of hypertension or the use of antihypertensive medication. Peripheral vascular disease (PVD) was defined as aorta and arteries other than coronary arteries with exercise-related claudication, reduced or absent pulsation or angiographic stenosis of more than $50 \%$.

Follow-up and endpoint event

In January 2021, all patients were followed up by trained professionals who were blinded to the baseline information. Information about adverse prognostic events was obtained from patients or their family members by telephone questionnaire. The information was further confirmed by careful verification of corresponding medical records if necessary. The primary observational endpoint was defined as MACCE consisting of four events as follows: all-cause death, nonfatal myocardial infarction (MI), nonfatal stroke and symptomatic graft stenosis or occlusion. The secondary observational endpoint was each component of the composite primary endpoint. MI was defined as elevated cardiac troponin higher than the upper reference limit with ischemia indicated by symptoms and/or electrocardiographic changes with or without an elevation of the ST segment. Symptomatic graft stenosis or occlusion was defined as having typical angina symptoms again after discharge and identification of graft stenosis or occlusion by coronary CTA scan. The first primary endpoint event that occurred during the follow-up was used for analysis in the current study. For patients with multiple adverse outcomes occurring almost simultaneously during the follow-up, only the most severe event (all-cause death $>$ nonfatal $\mathrm{MI}>$ nonfatal stroke > symptomatic graft stenosis or occlusion) was selected for the analyses. If the same event occurred multiple times, only the first occurrence was used for the analyses.

\section{Statistical analysis}

Continuous variables are presented as the mean \pm standard deviation (SD) or median (25th and 75th percentiles: P25, P75) in the case of normal or nonnormal distribution, and differences between the two groups were examined by independent-sample t-test or Mann-Whitney $U$ test correspondingly. Categorical variables are described as counts (percentages) and compared by Pearson chi-square test (Pearson $\chi^{2}$ test) with Yates' continuity correction. Spearman's rank correlation test or Pearson correlation test was used to evaluate the correlations between the TyG index and cardiovascular and cerebrovascular risk factors when appropriate. The Pearson correlation test was used to evaluate the correlation between two continuous variables with a normal distribution, while Spearman's rank correlation test was applied in cases where one or more of the variables being analyzed were nonnormally distributed continuous variables or categorical variables. Receiver operating characteristic (ROC) curve analysis was performed to determine the optimal cutoff point value of the TyG index for predicting the primary endpoint. KaplanMeier survival analyses were performed to evaluate the incidence rate of adverse events between groups 
according to the optimal cutoff point of the TyG index, and discrepancies between groups were evaluated by the log-rank test. The predictive value of the variables for the primary endpoint was evaluated by univariate and multivariate Cox proportional hazards analyses. The TyG index was analyzed in the following two ways: (1) as a categorical variable and (2) as a continuous variable. In the multivariate Cox proportional hazards analyses, four models were established to evaluate the predictive value of the TyG index for primary endpoint. Among these models, confounders were selected according to statistical significance $(P<0.2)$ in the univariate analysis and clinical importance. The models were as follows: Model 1, adjusted for age, sex (female), BMI, current smoking status, current drinking status, dyslipidemia, prior MI, prior PCl, prior stroke and PVD; Model 2, adjusted for variables included in Model 1 in addition to diagnosis (NSTEMI), TC, HDL-C, hs-CRP, creatinine, eGFR, uric acid, GA and LVEF; Model 3 , adjusted for variables included in Model 2 in addition to multiple grafts, LIMA use and IABP use; and Model 4, adjusted for variables included in Model 3 in addition to DAPT at discharge, statin at discharge, oral hypoglycemic agents (metformin, alpha-glucosidase inhibitor, sulfonylurea or dipeptidyl peptidase 4 inhibitor) at discharge and insulin at discharge. Further stratified analyses according to age ( $\leq 65$ and $>$ 65 years), sex, BMI ( $\leq 28$ and $>28 \mathrm{~kg} / \mathrm{m}^{2}$ ), hypertension, initial diagnosis (UA and NSTEMI), LDL-C ( $\leq 70$ and $>70 \mathrm{mg} / \mathrm{dL}$ ), LVEF ( $\leq 40 \%$ and $>40 \%$ ), medication at discharge including statins, oral hypoglycemic agents, insulin, type of grafts (LIMA- and LIMA+), number of grafts (one or two grafts and three or more grafts) and IAPB use were employed to examine the consistency of the prognostic impact of TyG index for primary endpoint. The model used in the stratified analyses consisted of all covariates used in Model 4 , except for the variables that were used for stratification. The interaction of the TyG index and variables used for stratification was examined by likelihood ratio tests.

The prognostic impact of the TyG index for each component of the primary endpoint was also assessed by using Model 4. FPG and TGs were not included in the multivariate analysis because they were used to calculate the TyG index. The results of Cox proportional hazards analyses are presented as hazard ratios (HRs) and $95 \%$ confidence intervals (Cls).

Statistical tests were performed with the R Programming Language (version 4.0.5). A two-tailed $\mathrm{P}$ value $<$ 0.05 was regarded as statistically significant.

\section{Results}

Baseline characteristics of patients

A total of 1534 admitted hospital patients were included with a median follow-up time of 23.1 months (interquartile range, 17.4-29.5 years) and a mean age of $62.7 \pm 8.2$ years old. Patients who experienced events had the following characteristics: older; higher levels of TGs, TC, LDL-C, eGFR, GA, Creatinine, FPG and TyG index; more likely to use multiple SVGs rather than LIMA + SVGs; and more likely to have lipidregulating agents than those who had no events (Table 1). Left ventricular ejection fraction, IABP support and medication at discharge were similar between these two groups. 
Table 1

Baseline clinical and laboratory characteristics of the study patients stratified by the primary endpoint

\begin{tabular}{|c|c|c|c|c|}
\hline & $\begin{array}{l}\text { Total population }(n= \\
\text { 1534) }\end{array}$ & $\begin{array}{l}\text { Without event(n = } \\
\text { 1370) }\end{array}$ & $\begin{array}{l}\text { With event(n = } \\
\text { 164) }\end{array}$ & $\begin{array}{l}\mathrm{P} \\
\text { value }\end{array}$ \\
\hline Age, years & $62.7 \pm 8.2$ & $62.4 \pm 8.1$ & $64.8 \pm 8.4$ & $\dot{0.001}$ \\
\hline Sex, male, n (\%) & $1092(71.2)$ & 981 (71.6) & $111(67.7)$ & 0.339 \\
\hline $\mathrm{BMI}, \mathrm{kg} / \mathrm{m}^{2}$ & $25.91 \pm 3.13$ & $25.88 \pm 3.10$ & $26.13 \pm 3.38$ & 0.347 \\
\hline SBP, mmHg & $130.63 \pm 16.63$ & $130.66 \pm 16.79)$ & $130.38 \pm 15.34$ & 0.836 \\
\hline $\mathrm{DBP}, \mathrm{mmHg}$ & $74.44 \pm 10.64$ & $74.47 \pm 10.69)$ & $74.15 \pm 10.23)$ & 0.715 \\
\hline Current smoking, n (\%) & $430(28.0)$ & $392(28.6)$ & $38(23.2)$ & 0.169 \\
\hline Current drinking, n (\%) & $314(20.5)$ & $290(21.2)$ & $24(14.6)$ & 0.063 \\
\hline \multicolumn{5}{|l|}{ Medical History, n (\%) } \\
\hline Hypertension & $1114(72.6)$ & 996 (72.7) & $118(72.0)$ & 0.912 \\
\hline Dyslipidemia & $483(31.5)$ & $415(30.3)$ & $68(41.5)$ & 0.005 \\
\hline Prior MI & $221(14.4)$ & $196(14.3)$ & $25(15.2)$ & 0.837 \\
\hline Prior PCl & $360(23.5)$ & 314 (22.9) & $46(28.0)$ & 0.172 \\
\hline Prior stroke & $160(10.4)$ & $145(10.6)$ & $15(9.1)$ & 0.664 \\
\hline PVD & 587 (38.3) & 505 (36.9) & $82(50.0)$ & 0.001 \\
\hline \multicolumn{5}{|l|}{ Laboratory results } \\
\hline TGs, mg/dL & $\begin{array}{l}128.56[94.87 \\
174.44]\end{array}$ & $\begin{array}{l}127.67[93.09 \\
170.23]\end{array}$ & $\begin{array}{l}139.64[104.40, \\
224.31]\end{array}$ & $\dot{0} .001$ \\
\hline $\mathrm{TC}, \mathrm{mg} / \mathrm{dL}$ & $146.54(36.91)$ & $145.52 \pm 35.88$ & $155.00 \pm 43.78$ & 0.002 \\
\hline LDL-C, mg/dL & $86.72 \pm 31.10$ & $86.14 \pm 30.43$ & $91.53 \pm 35.95$ & 0.036 \\
\hline $\mathrm{HDL}-\mathrm{C}, \mathrm{mg} / \mathrm{dL}$ & $37.40 \pm 8.44$ & $37.40 \pm 8.31$ & $37.41 \pm 9.49$ & 0.987 \\
\hline
\end{tabular}

Italic values indicate statistically significant associations

BMI body mass index, SBP systolic blood pressure, DBP diastolic blood pressure, MI myocardial infarction, PCl percutaneous coronary intervention, PVD peripheral vascular disease, TGs triglycerides, TC total cholesterol, LDL-C low-density lipoprotein cholesterol, HDL-C high-density lipoprotein cholesterol, hs-CRP high-sensitivity C-reactive protein, eGFR estimated glomerular filtration rate, FPG fasting plasma glucose, GA glycated albumin, TyG triglyceride glucose, LVEF left ventricular ejection fraction, UA unstable angina, NSTEMI non-ST-segment elevation myocardial infarction, LIMA left internal mammary artery, SVG saphenous vein grafts, ACEl angiotensin converting enzyme inhibitor, ARB angiotensin receptor blocker, DAPT dual antiplatelet therapy, DPP-4 Dipeptidyl peptidase-4, IABP intra-aortic balloon pump 


\begin{tabular}{|c|c|c|c|c|}
\hline & $\begin{array}{l}\text { Total population }(n= \\
\text { 1534) }\end{array}$ & $\begin{array}{l}\text { Without event }(n= \\
1370)\end{array}$ & $\begin{array}{l}\text { With event }(n= \\
\text { 164) }\end{array}$ & $\begin{array}{l}P \\
\text { value }\end{array}$ \\
\hline Hs-CRP, mg/L & $3.12 \pm 4.21$ & $3.09 \pm 4.20$ & $3.32 \pm 4.30$ & 0.511 \\
\hline Creatinine, $\mu \mathrm{mol} / \mathrm{L}$ & $75.53 \pm 46.57$ & $74.54 \pm 41.15$ & $83.82 \pm 78.11$ & 0.016 \\
\hline $\begin{array}{l}\text { eGFR, } \\
\mathrm{mL} /\left(\mathrm{min}^{\star} 1.73 \mathrm{~cm}^{2}\right)\end{array}$ & $91.16 \pm 16.49$ & $91.67 \pm 16.15$ & $86.89 \pm 18.61$ & $<.001$ \\
\hline Urid acid, $\mu \mathrm{mol} / \mathrm{L}$ & $331.19 \pm 92.22$ & $329.92 \pm 89.78$ & $341.74 \pm 110.32$ & 0.121 \\
\hline $\mathrm{FPG}, \mathrm{mg} / \mathrm{dL}$ & $\begin{array}{l}\text { 135.36 [111.46, } \\
174.56]\end{array}$ & $\begin{array}{l}\text { 133.83 [110.74, } \\
170.73]\end{array}$ & $\begin{array}{l}\text { 155.34 [120.82, } \\
208.62]\end{array}$ & ¿.001 \\
\hline GA, \% & $18.88 \pm 6.22$ & $18.74 \pm 6.16$ & $20.05 \pm 6.58$ & 0.011 \\
\hline TyG index & $9.13 \pm 0.63$ & $9.09 \pm 0.61$ & $9.40 \pm 0.66$ & $<.001$ \\
\hline LVEF, \% & $59.52 \pm 7.69$ & $59.61 \pm 7.62$ & $58.77 \pm 8.22$ & 0.185 \\
\hline \multicolumn{5}{|l|}{ Initial diagnosis, n (\%) } \\
\hline UA & $1451(94.6)$ & $1302(95.0)$ & 149 (90.9) & 0.040 \\
\hline NSTEMI & $83(5.4)$ & $68(5.0)$ & $15(9.1)$ & 0.040 \\
\hline \multicolumn{5}{|l|}{ Number of bridges } \\
\hline Only one bridge, n (\%) & $135(8.8)$ & $117(8.5)$ & $18(11.0)$ & 0.371 \\
\hline Two bridges, n (\%) & $500(32.6)$ & $454(33.1)$ & $46(28.0)$ & 0.220 \\
\hline Multiple bridges, n (\%) & $899(58.6)$ & 799 (58.3) & $100(61.0)$ & 0.570 \\
\hline \multicolumn{5}{|l|}{ Bridge type, n (\%) } \\
\hline Single LIMA & $126(8.2)$ & $112(8.2)$ & $14(8.5)$ & 0.993 \\
\hline Single SVG & $8(0.5)$ & $4(0.3)$ & $4(2.4)$ & 0.002 \\
\hline LIMA + SVGs & $951(62.0)$ & 875 (63.9) & $76(46.3)$ & $<.001$ \\
\hline
\end{tabular}

Italic values indicate statistically significant associations

BMI body mass index, SBP systolic blood pressure, DBP diastolic blood pressure, MI myocardial infarction, $\mathrm{PCl}$ percutaneous coronary intervention, PVD peripheral vascular disease, TGs triglycerides, TC total cholesterol, LDL-C low-density lipoprotein cholesterol, HDL-C high-density lipoprotein cholesterol, hs-CRP high-sensitivity C-reactive protein, eGFR estimated glomerular filtration rate, FPG fasting plasma glucose, GA glycated albumin, TyG triglyceride glucose, LVEF left ventricular ejection fraction, UA unstable angina, NSTEMI non-ST-segment elevation myocardial infarction, LIMA left internal mammary artery, SVG saphenous vein grafts, ACEI angiotensin converting enzyme inhibitor, ARB angiotensin receptor blocker, DAPT dual antiplatelet therapy, DPP-4 Dipeptidyl peptidase-4, IABP intra-aortic balloon pump 


\begin{tabular}{|c|c|c|c|c|}
\hline & $\begin{array}{l}\text { Total population }(\mathrm{n}= \\
\text { 1534) }\end{array}$ & $\begin{array}{l}\text { Without event( } \mathrm{n}= \\
\text { 1370) }\end{array}$ & $\begin{array}{l}\text { With event( } n= \\
\text { 164) }\end{array}$ & $\begin{array}{l}\mathrm{P} \\
\text { value }\end{array}$ \\
\hline Multiple SVGs & $446(29.1)$ & $376(27.4)$ & 70 (42.7) & $\stackrel{<}{0.001}$ \\
\hline \multicolumn{5}{|l|}{$\begin{array}{l}\text { Medication at } \\
\text { discharge, n (\%) }\end{array}$} \\
\hline ACEI or ARB & $30(2.0)$ & $24(1.8)$ & $6(3.7)$ & 0.171 \\
\hline DAPT & $1267(82.6)$ & $1131(82.6)$ & $136(82.9)$ & 0.992 \\
\hline Aspirin & $1463(95.4)$ & 1305 (95.3) & 158 (96.3) & 0.668 \\
\hline P2Y12R inhibitor & $1288(84.0)$ & $1148(83.8)$ & $140(85.4)$ & 0.685 \\
\hline$\beta$-blocker & $1120(73.0)$ & $1004(73.3)$ & 116 (70.7) & 0.547 \\
\hline Lipid regulating agents & $1400(91.3)$ & $1261(92.0)$ & 139 (84.8) & 0.003 \\
\hline $\begin{array}{l}\text { Oral hypoglycemic } \\
\text { agents }\end{array}$ & $629(41.0)$ & $566(41.3)$ & $63(38.4)$ & 0.529 \\
\hline Metformin & $422(27.5)$ & 380 (27.7) & $42(25.6)$ & 0.628 \\
\hline $\begin{array}{l}\text { Alpha-glucosidase } \\
\text { inhibitor }\end{array}$ & $422(27.5)$ & $372(27.2)$ & $50(30.5)$ & 0.417 \\
\hline Sulfonylurea & $18(1.2)$ & $17(1.2)$ & $1(0.6)$ & 0.745 \\
\hline DPP-4 & $17(1.1)$ & $15(1.1)$ & $2(1.2)$ & 1.000 \\
\hline Insulin & $49(3.2)$ & $43(3.1)$ & $6(3.7)$ & 0.902 \\
\hline \multicolumn{5}{|l|}{ Instrument assistance } \\
\hline IABP use, n (\%) & $27(1.8)$ & $23(1.7)$ & $4(2.4)$ & 0.700 \\
\hline \multicolumn{5}{|c|}{ Italic values indicate statistically significant associations } \\
\hline \multicolumn{5}{|c|}{$\begin{array}{l}\text { BMI body mass index, SBP systolic blood pressure, DBP diastolic blood pressure, MI myocardial } \\
\text { infarction, PCI percutaneous coronary intervention, PVD peripheral vascular disease, TGs triglycerides, } \\
\text { TC total cholesterol, LDL-C low-density lipoprotein cholesterol, HDL-C high-density lipoprotein } \\
\text { cholesterol, hs-CRP high-sensitivity C-reactive protein, eGFR estimated glomerular filtration rate, FPG } \\
\text { fasting plasma glucose, GA glycated albumin, TyG triglyceride glucose, LVEF left ventricular ejection } \\
\text { fraction, UA unstable angina, NSTEMI non-ST-segment elevation myocardial infarction, LIMA left } \\
\text { internal mammary artery, SVG saphenous vein grafts, ACEI angiotensin converting enzyme inhibitor, } \\
\text { ARB angiotensin receptor blocker, DAPT dual antiplatelet therapy, DPP-4 Dipeptidyl peptidase-4, IABP } \\
\text { intra-aortic balloon pump }\end{array}$} \\
\hline
\end{tabular}

ROC curve analysis showed that the AUC of the TyG index for predicting the primary endpoint was 0.631 (95\% Cl 0.584-0.677, P<0.001). The optimal cutoff point of the TyG index was 9.42 with a sensitivity of $49.4 \%$ and a specificity of $71.2 \%$. Patients with a higher TyG index were younger, had significantly higher BMl levels and had a higher proportion of dyslipidemia. Laboratory indexes, including TG, TC, LDL-C, hs- 
CRP, uric acid, FPG and GA, were significantly higher in patients with a higher TyG index, while HDL-C levels were relatively lower in these patients. However, Creatinine and eGFR, which were different between patients with and without events, were not significant between lower and higher TyG groups. In the higher TyG index group, more patients were diagnosed with NSTEMI (Table 2). Left ventricular ejection fraction, IABP support and medication at discharge were also similar between these two groups. 
Table 2

Baseline clinical characteristics of the study patients according to the cut-off value of TyG index

\begin{tabular}{|c|c|c|c|c|}
\hline & $\begin{array}{l}\text { Total population } \\
(n=1534)\end{array}$ & $\begin{array}{l}\text { Lower TyG index } \\
(\leq 9.42 ; n=745)\end{array}$ & $\begin{array}{l}\text { Higher TyG index } \\
(>9.42 ; n=359)\end{array}$ & $\begin{array}{l}P \\
\text { value }\end{array}$ \\
\hline Age, years & $62.68 \pm 8.19$ & $63.30 \pm 7.77$ & $61.31 \pm 8.91$ & $\dot{0} .001$ \\
\hline Sex, male, n (\%) & $442(28.8)$ & 760 (71.9) & $332(69.6)$ & 0.390 \\
\hline $\mathrm{BMI}, \mathrm{kg} / \mathrm{m}^{2}$ & $25.91 \pm 3.13$ & $25.59 \pm 3.01$ & $26.61 \pm 3.28$ & $\dot{0.001}$ \\
\hline SBP, mmHg & $130.63 \pm 16.63$ & $130.97 \pm 16.96$ & $129.89 \pm 15.87$ & 0.238 \\
\hline DBP, mmHg & $74.44 \pm 10.64$ & $74.11 \pm 10.89$ & $75.16 \pm 10.02$ & 0.074 \\
\hline Current smoking, n (\%) & $430(28.0)$ & $287(27.2)$ & $143(30.0)$ & 0.280 \\
\hline Current drinking, n (\%) & $314(20.5)$ & $214(20.2)$ & $100(21.0)$ & 0.799 \\
\hline \multicolumn{5}{|l|}{ Medical History, n (\%) } \\
\hline Hypertension & $1114(72.6)$ & 769 (72.8) & 345 (72.3) & 0.911 \\
\hline Dyslipidemia & $483(31.5)$ & $315(29.8)$ & $168(35.2)$ & 0.040 \\
\hline Prior MI & $221(14.4)$ & $148(14.0)$ & $73(15.3)$ & 0.553 \\
\hline Prior PCl & $360(23.5)$ & $234(22.1)$ & $126(26.4)$ & 0.078 \\
\hline Prior stroke & $160(10.4)$ & $114(10.8)$ & $46(9.6)$ & 0.557 \\
\hline PVD & $587(38.3)$ & 397 (37.6) & $190(39.8)$ & 0.429 \\
\hline \multicolumn{5}{|l|}{ Laboratory results } \\
\hline $\mathrm{TG}, \mathrm{mg} / \mathrm{dL}$ & $\begin{array}{l}128.56[94.87 \\
174.44]\end{array}$ & $\begin{array}{l}108.17[84.23 \\
134.76]\end{array}$ & $\begin{array}{l}207.46[157.81 \\
266.87]\end{array}$ & $\dot{0} .001$ \\
\hline $\mathrm{TC}, \mathrm{mg} / \mathrm{dL}$ & $146.54(36.91)$ & $140.68(34.73)$ & 159.53 (38.28) & $<.001$ \\
\hline
\end{tabular}

Italic values indicate statistically significant associations

BMI body mass index, SBP systolic blood pressure, DBP diastolic blood pressure, MI myocardial infarction, $\mathrm{PCl}$ percutaneous coronary intervention, PVD peripheral vascular disease, TGs triglycerides, TC total cholesterol, LDL-C low-density lipoprotein cholesterol, HDL-C high-density lipoprotein cholesterol, hs-CRP high-sensitivity C-reactive protein, eGFR estimated glomerular filtration rate, FPG fasting plasma glucose, GA glycated albumin, TyG triglyceride glucose, LVEF left ventricular ejection fraction, UA unstable angina, NSTEMI non-ST-segment elevation myocardial infarction, LIMA left internal mammary artery, SVG saphenous vein grafts, ACEl angiotensin converting enzyme inhibitor, ARB angiotensin receptor blocker, DAPT dual antiplatelet therapy, DPP-4 Dipeptidyl peptidase-4, IABP intra-aortic balloon pump 


\begin{tabular}{|c|c|c|c|c|}
\hline & $\begin{array}{l}\text { Total population } \\
(n=1534)\end{array}$ & $\begin{array}{l}\text { Lower TyG index } \\
(\leq 9.42 ; n=745)\end{array}$ & $\begin{array}{l}\text { Higher TyG index } \\
(>9.42 ; n=359)\end{array}$ & $\begin{array}{l}P \\
\text { value }\end{array}$ \\
\hline LDL-C, mg/dL & $86.72(31.10)$ & $83.91(29.87)$ & $92.94(32.85)$ & $\dot{0} 001$ \\
\hline $\mathrm{HDL}-\mathrm{C}, \mathrm{mg} / \mathrm{dL}$ & $37.40(8.44)$ & $38.56(8.73)$ & $34.83(7.12)$ & $\dot{0.001}$ \\
\hline Hs-CRP, mg/L & $3.12(4.21)$ & $2.95(4.07)$ & $3.50(4.49)$ & 0.018 \\
\hline Creatinine, $\mu \mathrm{mol} / \mathrm{L}$ & $75.53(46.57)$ & 74.07 (41.68) & $78.77(55.82)$ & 0.067 \\
\hline $\begin{array}{l}\text { eGFR, } \\
\mathrm{mL} /\left(\mathrm{min}^{\star} 1.73 \mathrm{~cm}^{2}\right)\end{array}$ & $91.16(16.49)$ & 91.68 (15.73) & $90.00(18.02)$ & 0.064 \\
\hline Uric acid, $\mu \mathrm{mol} / \mathrm{L}$ & 331.19 (92.22) & 324.60 (88.09) & 345.79 (99.33) & $\dot{0} .001$ \\
\hline $\mathrm{FPG}, \mathrm{mg} / \mathrm{dL}$ & $\begin{array}{l}\text { 135.36 [111.46, } \\
174.56]\end{array}$ & $\begin{array}{l}123.84[105.12, \\
146.16]\end{array}$ & $\begin{array}{l}181.26[147.60, \\
228.06]\end{array}$ & <. \\
\hline $\mathrm{GA}, \%$ & $18.88(6.22)$ & $18.45(5.96)$ & 19.85 (6.65) & $\begin{array}{l}< \\
0.001\end{array}$ \\
\hline TyG index & $9.13 \pm 0.63$ & $8.80 \pm 0.42$ & $9.85 \pm 0.33$ & $\dot{0} 001$ \\
\hline LVEF, \% & $59.52 \pm 7.69$ & $59.63 \pm 7.57$ & $59.27 \pm 7.95$ & 0.400 \\
\hline \multicolumn{5}{|l|}{ Initial diagnosis, n (\%) } \\
\hline UA & $1451(94.6)$ & $1010(95.6)$ & 441 (92.5) & 0.018 \\
\hline NSTEMI & $83(5.4)$ & $47(4.4)$ & $36(7.5)$ & 0.018 \\
\hline \multicolumn{5}{|l|}{ Number of bridges } \\
\hline Single bridge, n (\%) & $135(8.8)$ & $95(9.0)$ & $40(8.4)$ & 0.773 \\
\hline Two bridges, n (\%) & $500(32.6)$ & $333(31.5)$ & $167(35.0)$ & 0.195 \\
\hline Multiple bridges, n (\%) & $899(58.6)$ & $629(59.5)$ & $270(56.6)$ & 0.311 \\
\hline
\end{tabular}

Italic values indicate statistically significant associations

BMI body mass index, SBP systolic blood pressure, DBP diastolic blood pressure, MI myocardial infarction, $\mathrm{PCl}$ percutaneous coronary intervention, PVD peripheral vascular disease, TGs triglycerides, TC total cholesterol, LDL-C low-density lipoprotein cholesterol, HDL-C high-density lipoprotein cholesterol, hs-CRP high-sensitivity C-reactive protein, eGFR estimated glomerular filtration rate, FPG fasting plasma glucose, GA glycated albumin, TyG triglyceride glucose, LVEF left ventricular ejection fraction, UA unstable angina, NSTEMI non-ST-segment elevation myocardial infarction, LIMA left internal mammary artery, SVG saphenous vein grafts, ACEl angiotensin converting enzyme inhibitor, ARB angiotensin receptor blocker, DAPT dual antiplatelet therapy, DPP-4 Dipeptidyl peptidase-4, IABP intra-aortic balloon pump 


\section{Total population Lower TyG index Higher TyG index \\ $(n=1534) \quad(\leq 9.42 ; n=745) \quad(>9.42 ; n=359)$ \\ value}

Type of bridge, $n(\%)$

\begin{tabular}{|lllll|}
\hline Single LIMA & $126(8.2)$ & $89(8.4)$ & $37(7.8)$ & 0.736 \\
\hline Single SVG & $8(0.5)$ & $6(0.6)$ & $2(0.4)$ & 1.000 \\
\hline LIMA + SVGs & $951(62.0)$ & $654(61.9)$ & $297(62.3)$ & 0.929 \\
\hline Multiple SVGs & $446(29.1)$ & $307(29.0)$ & $139(29.1)$ & 1.000 \\
\hline $\begin{array}{l}\text { Medication at discharge, } \\
\text { n (\%) }\end{array}$ & & & & \\
\hline ACEl or ARB & $30(2.0)$ & $19(1.8)$ & $11(2.3)$ & 0.641 \\
\hline DAPT & $1267(82.6)$ & $858(81.2)$ & $409(85.7)$ & 0.035 \\
\hline Aspirin & $1463(95.4)$ & $1002(94.8)$ & $461(96.6)$ & 0.143 \\
\hline P2Y12R inhibitor & $1288(84.0)$ & $877(83.0)$ & $411(86.2)$ & 0.133 \\
\hline $\begin{array}{l}\text { B-blocker } \\
\text { Statin }\end{array}$ & $1120(73.0)$ & $768(72.7)$ & $352(73.8)$ & 0.688 \\
\hline $\begin{array}{l}\text { Oral hypoglycemic } \\
\text { agents }\end{array}$ & $1400(91.3)$ & $970(91.8)$ & $430(90.1)$ & 0.345 \\
\hline Metformin & $629(41.0)$ & $430(40.7)$ & $199(41.7)$ & 0.744 \\
\hline $\begin{array}{l}\text { Alpha-glucosidase } \\
\text { inhibitor }\end{array}$ & $422(27.5)$ & $287(27.2)$ & $135(28.3)$ & 0.686 \\
\hline $\begin{array}{l}\text { Sulfonylurea } \\
\text { DPP-4 }\end{array}$ & $18(1.2)$ & $288(27.2)$ & $134(28.1)$ & 0.778 \\
\hline $\begin{array}{l}\text { Insulin } \\
\text { Instrument assistance }\end{array}$ & $17(1.1)$ & $12(1.1)$ & $6(1.3)$ & $4(0.8)$ \\
\hline $49(3.2)$ & $13(1.2)$ & $19(4.0)$ & 0.679 \\
\hline
\end{tabular}

Italic values indicate statistically significant associations

BMI body mass index, SBP systolic blood pressure, DBP diastolic blood pressure, MI myocardial infarction, PCl percutaneous coronary intervention, PVD peripheral vascular disease, TGs triglycerides, TC total cholesterol, LDL-C low-density lipoprotein cholesterol, HDL-C high-density lipoprotein cholesterol, hs-CRP high-sensitivity C-reactive protein, eGFR estimated glomerular filtration rate, FPG fasting plasma glucose, GA glycated albumin, TyG triglyceride glucose, LVEF left ventricular ejection fraction, UA unstable angina, NSTEMI non-ST-segment elevation myocardial infarction, LIMA left internal mammary artery, SVG saphenous vein grafts, ACEI angiotensin converting enzyme inhibitor, ARB angiotensin receptor blocker, DAPT dual antiplatelet therapy, DPP-4 Dipeptidyl peptidase-4, IABP intra-aortic balloon pump 


\begin{tabular}{|c|c|c|c|c|}
\hline & $\begin{array}{l}\text { Total population } \\
(n=1534)\end{array}$ & $\begin{array}{l}\text { Lower TyG index } \\
(\leq 9.42 ; n=745)\end{array}$ & $\begin{array}{l}\text { Higher TyG index } \\
(>9.42 ; n=359)\end{array}$ & $\begin{array}{l}P \\
\text { value }\end{array}$ \\
\hline IABP use, $n(\%)$ & $27(1.8)$ & $17(1.6)$ & $10(2.1)$ & 0.643 \\
\hline \multicolumn{5}{|c|}{ Italic values indicate statistically significant associations } \\
\hline \multicolumn{5}{|c|}{$\begin{array}{l}\text { BMI body mass index, SBP systolic blood pressure, DBP diastolic blood pressure, MI myocardial } \\
\text { infarction, PCI percutaneous coronary intervention, PVD peripheral vascular disease, TGs triglycerides, } \\
\text { TC total cholesterol, LDL-C low-density lipoprotein cholesterol, HDL-C high-density lipoprotein } \\
\text { cholesterol, hs-CRP high-sensitivity C-reactive protein, eGFR estimated glomerular filtration rate, FPG } \\
\text { fasting plasma glucose, GA glycated albumin, TyG triglyceride glucose, LVEF left ventricular ejection } \\
\text { fraction, UA unstable angina, NSTEMI non-ST-segment elevation myocardial infarction, LIMA left } \\
\text { internal mammary artery, SVG saphenous vein grafts, ACEI angiotensin converting enzyme inhibitor, } \\
\text { ARB angiotensin receptor blocker, DAPT dual antiplatelet therapy, DPP-4 Dipeptidyl peptidase-4, IABP } \\
\text { intra-aortic balloon pump }\end{array}$} \\
\hline
\end{tabular}

Cardiovascular risk factors related to TyG index

Spearman's rank or Pearson correlation analysis was performed to determine the correlation between the TyG index and traditional or commonly used risk factors for cardiovascular disease.

The TyG index was positively correlated with sex (female), BMI, DBP, TGs, TC, LDL-C, hs-CRP, creatinine, uric acid, FPG, GA, diagnosis (NSTEMI) and dyslipidemia but negatively correlated with age, HDL-C and eGFR (Table 3). 
Table 3

Correlations between the TyG and traditional cardiovascular risk factors

Correlation coefficient

$-0.126$

0.054

0.197

0.008

0.079

0.797

0.291

0.178

$-0.254$

0.069

0.060

$-0.056$

0.105

0.638

0.124

$-0.025$

0.042

$-0.011$

0.068

0.029

0.047

$-0.037$

\section{$P$ value}

$<0.001$

0.036

$<0.001$

0.752

0.002

$<0.001$

$<0.001$

$<0.001$

$<0.001$

0.007

0.020

0.027

$<0.001$

$<0.001$

$<0.001$

0.331

0.104

0.677

0.008

0.258

prior $\mathrm{PCl}$

prior stroke

Italic values indicate statistically significant associations

BMI body mass index, SBP systolic blood pressure, DBP diastolic blood pressure, $\mathrm{PCl}$ percutaneous coronary intervention, PVD peripheral vascular disease, TGs triglycerides, TC total cholesterol, LDL-C low-density lipoprotein cholesterol, HDL-C high-density lipoprotein cholesterol, hs-CRP high-sensitivity C-reactive protein, eGFR estimated glomerular filtration rate, FPG fasting plasma glucose, GA glycated albumin, TyG triglyceride glucose, LVEF left ventricular ejection fraction, UA unstable angina, NSTEMI non-ST-segment elevation myocardial infarction, IABP intra-aortic balloon pump 


\begin{tabular}{|c|c|c|}
\hline & Correlation coefficient & $P$ value \\
\hline Prior PVD & 0.016 & 0.529 \\
\hline NSTEMI & 0.067 & 0.009 \\
\hline IABP & 0.044 & 0.088 \\
\hline multiple bridges & -0.024 & 0.348 \\
\hline hypertension & -0.017 & 0.506 \\
\hline \multicolumn{3}{|c|}{ Italic values indicate statistically significant associations } \\
\hline \multicolumn{3}{|c|}{$\begin{array}{l}\text { BMI body mass index, SBP systolic blood pressure, DBP diastolic blood pressure, } \mathrm{PCl} \text { percutaneous } \\
\text { coronary intervention, PVD peripheral vascular disease, TGs triglycerides, TC total cholesterol, LDL-C } \\
\text { low-density lipoprotein cholesterol, HDL-C high-density lipoprotein cholesterol, hs-CRP high-sensitivity } \\
\text { C-reactive protein, eGFR estimated glomerular filtration rate, FPG fasting plasma glucose, GA glycated } \\
\text { albumin, TyG triglyceride glucose, LVEF left ventricular ejection fraction, UA unstable angina, NSTEMI } \\
\text { non-ST-segment elevation myocardial infarction, IABP intra-aortic balloon pump }\end{array}$} \\
\hline
\end{tabular}

Primary and secondary outcomes are all significantly different between the lower and higher TyG index groups

During the follow-up period, 164 (10.7\%) MACCEs, including 25 (1.6\%) all-cause deaths, 32 (0.2\%) nonfatal Mls, $30(0.2 \%)$ nonfatal strokes and 79 (5.1) symptomatic graft stenoses or occlusions, were documented and included in the present analyses. The incidence of the primary endpoint increased significantly in patients with a higher TyG index compared to those with a lower TyG index (all Chi-square $P<0.05$ ) (Table 4). Kaplan-Meier curves for the incidence of the primary endpoint and each component according to the optimal cutoff point of the TyG index are shown in Fig. 2. Primary endpoint, all-cause death, nonfatal $\mathrm{Ml}$, nonfatal stroke and symptomatic graft stenosis and occlusion all showed a significant difference between the lower and higher TyG index groups (Fig. 2; primary endpoint log-rank $P$ $<0.001$; all secondary endpoints log-rank $P<0.05$ ). 
Table 4

Incidence of endpoint events according to the optimal cutoff point of TyG index

\begin{tabular}{|c|c|c|c|c|}
\hline & $\begin{array}{l}\text { Total } \\
\text { population } \\
(n=1534)\end{array}$ & $\begin{array}{l}\text { Lower TyG } \\
\text { index } \\
(\leq 9.42 ; n= \\
745)\end{array}$ & $\begin{array}{l}\text { Higher TyG } \\
\text { index } \\
(>9.42 ; n= \\
359)\end{array}$ & $\begin{array}{l}\mathrm{P} \\
\text { value }\end{array}$ \\
\hline Primary endpoint, n (\%) & $164(10.7)$ & $83(7.9)$ & $81(17.0)$ & $\hat{0} .001$ \\
\hline All-cause death, $\mathrm{n}(\%)$ & $25(1.6)$ & $10(0.9)$ & $15(3.1)$ & 0.003 \\
\hline Non-fatal MI, n (\%) & $32(0.2)$ & $15(1.4)$ & $17(3.6)$ & 0.011 \\
\hline Non-fatal stroke, n (\%) & $30(0.2)$ & $15(1.4)$ & $15(3.1)$ & 0.039 \\
\hline $\begin{array}{l}\text { Symptomatic graft stenosis or } \\
\text { occlusion, } \mathrm{n}(\%)\end{array}$ & $79(5.1)$ & $44(4.2)$ & $35(7.3)$ & 0.013 \\
\hline \multicolumn{5}{|c|}{ The groups were stratified by the optimal cutoff point of TyG index determined by ROC curve analysis } \\
\hline \multicolumn{5}{|c|}{ Italic values indicate statistically significant associations } \\
\hline TyG triglyceride glucose, MI my & arction & & & \\
\hline
\end{tabular}

Cox proportional hazard analyses of the prognostic implication of the TyG index

In the multivariate Cox proportional hazard analysis, four models (Models 1-4 as described above) comprised of variables that had statistical significance $(P<0.2)$ and/or clinical importance were constructed to evaluate the predictive potential of the TyG index for the primary endpoint. After adjusting for confounding variables, a higher TyG index remained an independent risk predictor of the primary endpoint despite regarding the TyG index as a nominal or continuous variable (all $\mathrm{P}<0.001$ in Models 14) (Table 5). Detailed information on Model 4 is shown in Additional file 1: Table S1. 
Table 5

Predictive value of TyG index for primary endpoint in different Cox proportional hazards models

\begin{tabular}{|c|c|c|c|c|c|c|}
\hline \multirow{3}{*}{ Crude model } & \multicolumn{3}{|c|}{ TyG index as a continuous variable ${ }^{a}$} & \multicolumn{3}{|c|}{ TyG index as a nominal variable ${ }^{b}$} \\
\hline & \multirow{2}{*}{$\begin{array}{l}\text { HR } \\
2.218\end{array}$} & \multirow{2}{*}{$\begin{array}{l}95 \% \mathrm{Cl} \\
1.734-2.839\end{array}$} & \multirow{2}{*}{$\begin{array}{l}\text { P value } \\
<0.001\end{array}$} & \multirow{2}{*}{$\begin{array}{l}\text { HR } \\
2.381\end{array}$} & \multicolumn{2}{|c|}{$95 \% \mathrm{Cl} \quad \mathrm{P}$ value } \\
\hline & & & & & $1.752-3.234$ & $<0.001$ \\
\hline Model 1 & 2.265 & $1.757-2.918$ & $<0.001$ & 2.413 & $1.765-3.299$ & $<0.001$ \\
\hline Model 2 & 2.142 & $1.642-2.794$ & $<0.001$ & 2.212 & $1.597-3.063$ & $<0.001$ \\
\hline Model 3 & 2.109 & $1.616-2.752$ & $<0.001$ & 2.191 & $1.579-3.039$ & $<0.001$ \\
\hline Model 4 & 2.105 & $1.609-2.755$ & $<0.001$ & 2.193 & $1.577-3.049$ & $<0.001$ \\
\hline \multicolumn{7}{|c|}{$\begin{array}{l}\text { Model 1: adjusted for age, sex(female), BMI, DBP, current smoking status, current drinking status, } \\
\text { dyslipidemia, prior MI, prior PCl, prior stroke and PVD; }\end{array}$} \\
\hline \multicolumn{7}{|c|}{$\begin{array}{l}\text { Model 2: adjusted for variables included in Model } 1 \text { and diagnosis (NSTEMI), TC, HDL-C, hs-CRP, } \\
\text { creatinine, eGFR, Uric Acid, GA, LVEF; }\end{array}$} \\
\hline \multicolumn{7}{|c|}{ Model 3: adjusted for variables included in Model 2 and multiple bridges, LIMA use, IABP use; } \\
\hline \multicolumn{7}{|c|}{$\begin{array}{l}\text { Model 4: adjusted for variables included in Model } 3 \text { and DAPT at discharge, statin at discharge, oral } \\
\text { hypoglycemic agents (metformin, alpha-glucosidase inhibitor, sulfonylurea, DPP-4) at discharge and } \\
\text { insulin at discharge }\end{array}$} \\
\hline \multicolumn{7}{|c|}{ TyG triglyceride glucose, $\mathrm{HR}$ hazard ratio, $\mathrm{Cl}$ confidence interval } \\
\hline \multicolumn{7}{|c|}{$\begin{array}{l}\text { The HR was examined regarding lower TyG index as reference (stratified by the optimal cutoff point } \\
\text { of TyG index determined by ROC curve analysis) }\end{array}$} \\
\hline \multicolumn{7}{|c|}{ a The HR was examined by per 1 -unit increase of TyG index } \\
\hline
\end{tabular}

We used Model 4 to evaluate the predictive value of the TyG index for MACCEs and each component. The results showed that a 1-unit increase in the TyG index was independently associated with a higher risk of MACCEs [HR 2.218 (1.733-2.839), $\mathrm{P}<0.001$ ] and each component. A higher TyG index of more than 9.42 was independently associated with a higher risk of MACCEs [HR $2.092(1.573-2.784), P<0.001]$ and each component (Table 6). 
Table 6

Predictive value of TyG index for primary endpoint and each component in univariate and multivariate analysis

\begin{tabular}{|c|c|c|c|c|c|c|}
\hline & \multicolumn{3}{|c|}{ Univariate analysis } & \multicolumn{3}{|c|}{ Multivariate analysis $^{c}$} \\
\hline & HR & $95 \% \mathrm{Cl}$ & $\begin{array}{l}P \\
\text { value }\end{array}$ & HR & $95 \% \mathrm{Cl}$ & $\begin{array}{l}P \\
\text { value }\end{array}$ \\
\hline \multicolumn{7}{|l|}{$\begin{array}{l}\text { TyG index as a continuous } \\
\text { variable }^{\mathrm{a}}\end{array}$} \\
\hline Primary Endpoint & 2.218 & $\begin{array}{l}1.734- \\
2.839\end{array}$ & $<.001$ & 2.092 & $\begin{array}{l}1.573- \\
2.784\end{array}$ & $\stackrel{<}{0.001}$ \\
\hline All-cause Death & 2.913 & $\begin{array}{l}1.552- \\
5.467\end{array}$ & $<.001$ & 3.372 & $\begin{array}{l}1.552- \\
7.325\end{array}$ & 0.002 \\
\hline Non-fatal MI & 2.635 & $\begin{array}{l}1.509- \\
4.604\end{array}$ & $\begin{array}{l}< \\
0.001\end{array}$ & 2.427 & $\begin{array}{l}1.268- \\
4.644\end{array}$ & 0.007 \\
\hline Non-fatal Stroke & 1.964 & $\begin{array}{l}1.112- \\
3.469\end{array}$ & 0.020 & 2.022 & $\begin{array}{l}1.042- \\
3.925\end{array}$ & 0.038 \\
\hline $\begin{array}{l}\text { Symptomatic graft stenosis or } \\
\text { occlusion }\end{array}$ & 1.867 & $\begin{array}{l}1.314- \\
2.654\end{array}$ & $<0.001$ & 1.672 & $\begin{array}{l}1.100- \\
2.542\end{array}$ & 0.016 \\
\hline \multicolumn{7}{|l|}{ TyG index as a nominal variable ${ }^{b}$} \\
\hline Primary Endpoint & 2.381 & $\begin{array}{l}1.752- \\
3.234\end{array}$ & $<.001$ & 2.115 & $\begin{array}{l}1.501- \\
2.980\end{array}$ & $\dot{0} 001$ \\
\hline All-cause Death & 3.441 & $\begin{array}{l}1.545- \\
7.660\end{array}$ & 0.003 & 3.187 & $\begin{array}{l}1.281- \\
7.929\end{array}$ & 0.013 \\
\hline Non-fatal MI & 2.716 & $\begin{array}{l}1.356- \\
5.441\end{array}$ & 0.005 & 2.308 & $\begin{array}{l}1.035- \\
5.148\end{array}$ & 0.041 \\
\hline Non-fatal Stroke & 2.345 & $\begin{array}{l}1.146- \\
4.800\end{array}$ & 0.020 & 2.374 & $\begin{array}{l}1.068- \\
5.274\end{array}$ & 0.034 \\
\hline
\end{tabular}

Italic values indicate statistically significant associations

TyG triglyceride glucose, MI myocardial infarction, $\mathrm{HR}$ hazard ratio, $\mathrm{Cl}$ confidence interval

a The HR was examined by per 1-unit increase of TyG index

b The HR was examined regarding lower TyG index as reference (stratified by the optimal cutoff point of TyG index determined by ROC curve analysis)

${ }^{\mathrm{c}}$ The multivariate analysis was performed by using Model 4 [adjusted for age, sex (female), BMI, current smoking status, current drinking status, dyslipidemia, prior MI, prior PCl, prior stroke, PVD, diagnosis (NSTEMI), TC, HDL-C, hs-CRP, creatinine, eGFR, uric acid, GA, LVEF, multiple grafts, LIMA use and IABP use, DAPT at discharge, statin at discharge, oral hypoglycemic agents (metformin, alphaglucosidase inhibitor, sulfonylurea or dipeptidyl peptidase 4 inhibitor) at discharge and insulin at discharge. 


\begin{tabular}{|c|c|c|c|c|c|c|}
\hline & \multicolumn{3}{|c|}{ Univariate analysis } & \multicolumn{3}{|c|}{ Multivariate analysis ${ }^{c}$} \\
\hline & HR & $95 \% \mathrm{Cl}$ & $\begin{array}{l}P \\
\text { value }\end{array}$ & HR & $95 \% \mathrm{Cl}$ & $\begin{array}{l}P \\
\text { value }\end{array}$ \\
\hline $\begin{array}{l}\text { Symptomatic graft stenosis or } \\
\text { occlusion }\end{array}$ & 1.887 & $\begin{array}{l}1.210- \\
2.942\end{array}$ & 0.005 & 1.567 & $\begin{array}{l}0.950- \\
2.585\end{array}$ & 0.079 \\
\hline \multicolumn{7}{|c|}{ Italic values indicate statistically significant associations } \\
\hline \multicolumn{7}{|c|}{ TyG triglyceride glucose, MI myocardial infarction, HR hazard ratio, Cl confidence interval } \\
\hline \multicolumn{7}{|c|}{ a The HR was examined by per 1-unit increase of TyG index } \\
\hline \multicolumn{7}{|c|}{$\begin{array}{l}\text { b The HR was examined regarding lower TyG index as reference (stratified by the optimal cutoff point } \\
\text { of TyG index determined by ROC curve analysis) }\end{array}$} \\
\hline \multicolumn{7}{|c|}{$\begin{array}{l}\text { c The multivariate analysis was performed by using Model } 4 \text { [adjusted for age, sex (female), BMI, } \\
\text { current smoking status, current drinking status, dyslipidemia, prior MI, prior PCl, prior stroke, PVD, } \\
\text { diagnosis (NSTEMI), TC, HDL-C, hs-CRP, creatinine, eGFR, uric acid, GA, LVEF, multiple grafts, LIMA use } \\
\text { and IABP use, DAPT at discharge, statin at discharge, oral hypoglycemic agents (metformin, alpha- } \\
\text { glucosidase inhibitor, sulfonylurea or dipeptidyl peptidase } 4 \text { inhibitor) at discharge and insulin at } \\
\text { discharge. }\end{array}$} \\
\hline
\end{tabular}

Further evaluation of the risk stratification value of the TyG index for the primary endpoint was performed in various subgroups of the study population. The efficacy of TyG in increasing MACCE was consistent across all groups of age, sex, BMI, hypertension, LDL-C, oral hypoglycemic agents, LIMA, number of grafts and IABP groups (Fig. 3). Interestingly, the predictive value of the TyG index was prominent in patients who were supported by IABP perioperatively [HR $(95 \% \mathrm{CI})$ without IABP 1.731 (1.103-2.718) vs. with IABP 51.960 (6.338-425.959), $\mathrm{P}<0.001$ ] (Fig. 3).

\section{Discussion}

The primary finding of the present study was that an increased TyG index is a strong indicator of MACCEs, including all-cause death, nonfatal $\mathrm{MI}$ and stroke, and symptomatic graft stenosis or occlusion, in patients with NSTE-ACS and T2DM who underwent OPCABG, even after adjustment for confounding risk factors. Compared to participants with a lower TyG index, those with a higher TyG index had an apparently higher incidence of MACCEs and each component.

Insulin resistance (IR) is traditionally measured by the homeostatic model assessment of IR (HOMA-IR) [15]. However, it is inconvenient to measure insulin levels to calculate HOMA-IR. Several previous studies have suggested that the value of the TyG index for predicting IR is better than that of $\operatorname{HOMA-IR}[16,17]$. Compared to HOMA-IR, the TyG index does not require quantification of insulin but does require FPB and fasting TG. It is well known that an increased TyG index is associated with increased risks of T2DM and CVD $[16,18-20]$. Recently, several studies have found a significant correlation between the TyG index and clinical prognosis in patients with ACS. Qi Z. et al. found that the TyG index may be a better predictor of 
cardiovascular risk than FPG or HbA1C for patients with ACS undergoing PCI [21]. Chengping $\mathrm{H}$. et al. found that the TyG index may be a better predictor of cardiovascular risk than FPG or HbA1C for patients with ACS undergoing PCI [22]. Luo E. et al. also found that an increased TyG index may be an effective indicator of worse prognosis in patients with acute ST-segment elevation myocardial infarction (STEMI) who were treated with PCI for a 1-year follow-up [23]. To date, there are no reported studies on the TyG index and CABG to the best of our knowledge. We extend previous studies by proposing that the TyG index might be an effective indicator of the adverse prognosis of OPCABG in patients with T2DM and NSTE-ACS. Similarly, several studies have reported the cutoff values of the TyG index to be 9.18 and 9.323 [21, 24], while the cutoff value in the present study was 9.42. A TyG index higher than 9.42 indicates a higher risk of MACCEs after OPCABG in 2 years.

We also analyzed the predictive value by stratification according to the use of statins, oral hypoglycemic agents and insulin, but no significant difference was found. Unexpectedly, the predictive value of the TyG index was more significant in patients with perioperative IABP insertion compared to patients without IABP insertion, indicating that patients using IABPs are more sensitive to an increase in the TyG index. Thus, patients with IABP may have had more severe coronary disease and less coronary flow reserve [25], suggesting that little progress in treating coronary atherosclerosis may have a very large effect on heart function in the future.

The potential mechanism in the association of the TyG index with the development and progression of cardiovascular disease remains uncertain. Three potential hypotheses regarding the TyG index and the prognosis of CABG are detailed below. (1) Head SJ et al. found that the stroke rate of diabetic patients who underwent $\mathrm{CABG}$ is greater than that of patients who underwent $\mathrm{PCl}$ at the 5-year follow-up (CABG: $4.9 \%$ vs. PCl: $2.6 \%$ ) [26]. Domanski MJ found that renal insufficiency and baseline low-density lipoprotein $\geq 105 \mathrm{mg} / \mathrm{dL}$ are independent late stroke predictors [27]. In the present study, participants with a higher TyG index tended to have more severe and complex clinical conditions in terms of higher lipid profiles and lower eGFR. The correlation analysis also showed that the TyG index is positively related to multiple risk factors for LDL-C and negative for eGFR. (2) Many studies have shown that patients with diabetes who underwent CABG have a higher rate of myocardial infarction than those without diabetes. In the present study, among diabetic patients, the higher TyG group had a higher incidence of acute myocardial infarction and symptomatic graft stenosis or occlusion. A higher TyG index may affect native coronary atherosclerosis because the TyG index has been demonstrated to be related to coronary artery calcification [28] and arterial stiffness [29-31]. In addition, recent studies have found that IR is associated with coronary plaque vulnerability $[32,33]$. In contrast, a higher level of TyG may accelerate the atherosclerosis of grafts and accelerate the process of graft failure because IR has a proinflammatory and procoagulatory effect and is associated with endothelial dysfunction [34, 35]. (3) Low S et al. reported that FPG and TGs mainly reflect IR from the liver and adipose cells, respectively [7]. Therefore, the TyG index may reflect IR from these two aspects and thus be closely related to IR, which has been widely demonstrated to have a significant relationship with endothelial dysfunction, oxidative stress, cardiovascular remodeling, coagulation imbalance and inflammatory response, leading to late graft failure. 
This study had several limitations. (1) The study was a single-center, retrospective, observational study in a highly selected cohort with strict exclusion criteria, and the sample size was relatively small, which may have weakened the power of the results. Further prospective, multicenter studies in a more extensive population with a larger sample size are needed to verify the present findings. (2) The TyG index was assessed only once at admission. The changes in the TyG index during the follow-up period, which may have better predictive value for adverse prognosis, were not assessed in our study. (3) A certain proportion of participants received lipid-regulating therapy and antidiabetic treatment at admission, which may have had a potential impact on the TyG index and the study results. (4) Nearly all of the study population was Chinese patients. The results should be cautiously interpreted and expanded to Western populations as differences in metabolic levels exist among different races. (5) It is difficult to rule out that some patients may be complicated with undiagnosed systemic diseases, such as occult malignancies, which may have an impact on the assessment of prognosis. (6) We did not calculate the SYNTAX score and did not know whether complete revascularization occurred because most of the coronary angiograms were in optical discs from other hospitals, which prevented retrospective analysis. (7) The follow-up time was relatively short.

\section{Conclusions}

Based on retrospective studies, we concluded that the TyG index predicts future MACCEs in patients with T2DM and NSTE-ACS who underwent OPCABG independently of known cardiovascular risk factors. Importantly, this relationship remained significant after adjustment for other confounders, suggesting that the TyG index may be a useful marker for prognosis in patients with T2DM and NSTE-ACS who underwent OPCABG. Further prospective, large studies are required to confirm our findings.

\section{Declarations}

\section{Ethics approval and consent to participate}

This study was approved by the institutional review board of Beijing Anzhen Hospital, Capital Medical University. Given the retrospective nature of this study, the requirement for informed consent was waived.

\section{Availability of data and materials}

The datasets used during the current study are available from the corresponding author on reasonable request.

\section{Conflict of Interest}

The authors declare that the research was conducted in the absence of any commercial or financial relationships that could be construed as a potential conflict of interest. 


\section{Competing interests}

The authors declare that they have no competing interests.

\section{Funding}

This work was supported by the National Natural Science Foundation of China (NSFC) (81770343).

\section{Acknowledgments}

Not applicable.

\section{Author Contribution}

All authors were involved in the conception and design of the study and in the collection, analysis, and interpretation of the data. All authors reviewed the final manuscript. All authors read and approved the final manuscript.

\section{References}

1. Sousa-Uva M, Neumann FJ, Ahlsson A, Alfonso F, Banning AP, Benedetto U, Byrne RA, Collet JP, Falk V, Head SJ, et al. 2018 ESC/EACTS Guidelines on myocardial revascularization. Eur J Cardiothorac Surg. 2019;55(1):4-90.

2. Fox KA, Carruthers KF, Dunbar DR, Graham C, Manning JR, De Raedt H, Buysschaert I, Lambrechts D, Van de Werf F. Underestimated and under-recognized: the late consequences of acute coronary syndrome (GRACE UK-Belgian Study). Eur Heart J. 2010;31(22):2755-64.

3. Du T, Yuan G, Zhang M, Zhou X, Sun X, Yu X. Clinical usefulness of lipid ratios, visceral adiposity indicators, and the triglycerides and glucose index as risk markers of insulin resistance. Cardiovasc Diabetol. 2014;13:146.

4. Simental-Mendía LE, Rodríguez-Morán M, Guerrero-Romero F. The product of fasting glucose and triglycerides as surrogate for identifying insulin resistance in apparently healthy subjects. Metab Syndr Relat Disord. 2008;6(4):299-304.

5. Xie Y, Guo R, Li Z, Guo X, Sun G, Sun Z, Zheng J, Sun Y, Zheng L. Temporal relationship between body mass index and triglyceride-glucose index and its impact on the incident of hypertension. Nutr Metab Cardiovasc Dis. 2019;29(11):1220-9.

6. Liu EQ, Weng YP, Zhou AM, Zeng CL. Association between Triglyceride-Glucose Index and Type 2 Diabetes Mellitus in the Japanese Population: A Secondary Analysis of a Retrospective Cohort Study. Biomed Res Int. 2020;2020:2947067. 
7. Low S, Khoo KCJ, Irwan B, Sum CF, Subramaniam T, Lim SC, Wong TKM. The role of triglyceride glucose index in development of Type 2 diabetes mellitus. Diabetes Res Clin Pract. 2018;143:43-9.

8. Ma X, Dong L, Shao Q, Cheng Y, Lv S, Sun Y, Shen H, Wang Z, Zhou Y, Liu X. Triglyceride glucose index for predicting cardiovascular outcomes after percutaneous coronary intervention in patients with type 2 diabetes mellitus and acute coronary syndrome. Cardiovasc Diabetol. 2020;19(1):31.

9. Chiu TH, Tsai HJ, Chiou HC, Wu PY, Huang JC, Chen SC. A high triglyceride-glucose index is associated with left ventricular dysfunction and atherosclerosis. Int J Med Sci. 2021;18(4):1051-7.

10. Lambrinoudaki I, Kazani MV, Armeni E, Georgiopoulos G, Tampakis K, Rizos D, Augoulea A, Kaparos G, Alexandrou A, Stamatelopoulos K. The TyG Index as a Marker of Subclinical Atherosclerosis and Arterial Stiffness in Lean and Overweight Postmenopausal Women. Heart Lung Circ. 2018;27(6):716-24.

11. Alizargar J, Bai CH, Hsieh NC, Wu SV. Use of the triglyceride-glucose index (TyG) in cardiovascular disease patients. Cardiovasc Diabetol. 2020;19(1):8.

12. Levey AS, Coresh J, Greene T, Stevens LA, Zhang YL, Hendriksen S, Kusek JW, Van Lente F. Using standardized serum creatinine values in the modification of diet in renal disease study equation for estimating glomerular filtration rate. Ann Intern Med. 2006;145(4):247-54.

13. Alberti KG, Zimmet PZ. Definition, diagnosis and classification of diabetes mellitus and its complications. Part 1: diagnosis and classification of diabetes mellitus provisional report of a WHO consultation. Diabet Med. 1998;15(7):539-53.

14. Roffi M, Patrono C, Collet JP, Mueller C, Valgimigli M, Andreotti F, Bax JJ, Borger MA, Brotons C, Chew DP, et al. 2015 ESC Guidelines for the management of acute coronary syndromes in patients presenting without persistent ST-segment elevation: Task Force for the Management of Acute Coronary Syndromes in Patients Presenting without Persistent ST-Segment Elevation of the European Society of Cardiology (ESC). Eur Heart J. 2016;37(3):267-315.

15. Wallace TM, Matthews DR. The assessment of insulin resistance in man. Diabet Med. 2002;19(7):527-34.

16. Lee SH, Kwon HS, Park YM, Ha HS, Jeong SH, Yang HK, Lee JH, Yim HW, Kang MI, Lee WC, et al. Predicting the development of diabetes using the product of triglycerides and glucose: the Chungju Metabolic Disease Cohort (CMC) study. PLoS One. 2014;9(2):e90430.

17. Vasques AC, Novaes FS, de Oliveira Mda S, Souza JR, Yamanaka A, Pareja JC, Tambascia MA, Saad MJ, Geloneze B. TyG index performs better than HOMA in a Brazilian population: a hyperglycemic clamp validated study. Diabetes Res Clin Pract. 2011;93(3):e98-100.

18. Navarro-González D, Sánchez-Íñigo L, Pastrana-Delgado J, Fernández-Montero A, Martinez JA. Triglyceride-glucose index (TyG index) in comparison with fasting plasma glucose improved diabetes prediction in patients with normal fasting glucose: The Vascular-Metabolic CUN cohort. Prev Med. 2016;86:99-105.

19. Sánchez-Íñigo L, Navarro-González D, Fernández-Montero A, Pastrana-Delgado J, Martínez JA. The TyG index may predict the development of cardiovascular events. Eur J Clin Invest. 2016;46(2):189- 
97.

20. Li S, Guo B, Chen H, Shi Z, Li Y, Tian Q, Shi S. The role of the triglyceride (triacylglycerol) glucose index in the development of cardiovascular events: a retrospective cohort analysis. Sci Rep. 2019;9(1):7320.

21. Zhao Q, Zhang TY, Cheng YJ, Ma Y, Xu YK, Yang JQ, Zhou YJ. Impacts of triglyceride-glucose index on prognosis of patients with type 2 diabetes mellitus and non-ST-segment elevation acute coronary syndrome: results from an observational cohort study in China. Cardiovasc Diabetol. 2020;19(1):108.

22. Hu C, Zhang J, Liu J, Liu Y, Gao A, Zhu Y, Zhao Y. Discordance between the triglyceride glucose index and fasting plasma glucose or $\mathrm{HbA} 1 \mathrm{C}$ in patients with acute coronary syndrome undergoing percutaneous coronary intervention predicts cardiovascular events: a cohort study from China. Cardiovasc Diabetol. 2020;19(1):116.

23. Luo E, Wang D, Yan G, Qiao Y, Liu B, Hou J, Tang C. High triglyceride-glucose index is associated with poor prognosis in patients with acute ST-elevation myocardial infarction after percutaneous coronary intervention. Cardiovasc Diabetol. 2019;18(1):150.

24. Wang L, Cong HL, Zhang JX, Hu YC, Wei A, Zhang YY, Yang H, Ren LB, Qi W, Li WY, et al. Triglycerideglucose index predicts adverse cardiovascular events in patients with diabetes and acute coronary syndrome. Cardiovasc Diabetol. 2020;19(1):80.

25. Rubino AS, Onorati F, Scalas C, Serraino GF, Marsico R, Gelsomino S, Lorusso R, Renzulli A. Intraaortic balloon pumping recruits graft flow reserve by lowering coronary resistances. Int $\mathrm{J}$ Cardiol. 2012;154(3):293-8.

26. Head SJ, Milojevic M, Daemen J, Ahn JM, Boersma E, Christiansen EH, Domanski MJ, Farkouh ME, Flather M, Fuster V, et al. Stroke Rates Following Surgical Versus Percutaneous Coronary Revascularization. J Am Coll Cardiol. 2018;72(4):386-98.

27. Domanski MJ, Farkouh ME, Zak V, Feske S, Easton D, Weinberger J, Hamon M, Escobedo J, Shrader P, Siami FS, et al. Predictors of stroke associated with coronary artery bypass grafting in patients with diabetes mellitus and multivessel coronary artery disease. Am J Cardiol. 2015;115(10):1382-8.

28. Won KB, Park EJ, Han D, Lee JH, Choi SY, Chun EJ, Park SH, Han HW, Sung J, Jung HO, et al. Triglyceride glucose index is an independent predictor for the progression of coronary artery calcification in the absence of heavy coronary artery calcification at baseline. Cardiovasc Diabetol. 2020;19(1):34.

29. Fiorentino TV, Marini MA, Succurro E, Andreozzi F, Sesti G. Relationships of surrogate indexes of insulin resistance with insulin sensitivity assessed by euglycemic hyperinsulinemic clamp and subclinical vascular damage. BMJ Open Diabetes Res Care. 2019;7(1):e000911.

30. Zhao S, Yu S, Chi C, Fan X, Tang J, Ji H, Teliewubai J, Zhang Y, Xu Y. Association between macroand microvascular damage and the triglyceride glucose index in community-dwelling elderly individuals: the Northern Shanghai Study. Cardiovasc Diabetol. 2019;18(1):95.

31. Nakagomi A, Sunami Y, Kawasaki Y, Fujisawa T, Kobayashi Y. Sex difference in the association between surrogate markers of insulin resistance and arterial stiffness. J Diabetes Complications. 
2020;34(6):107442.

32. Iguchi T, Hasegawa T, Otsuka K, Matsumoto K, Yamazaki T, Nishimura S, Nakata S, Ehara S, Kataoka $\mathrm{T}$, Shimada $\mathrm{K}$, et al. Insulin resistance is associated with coronary plaque vulnerability: insight from optical coherence tomography analysis. Eur Heart J Cardiovasc Imaging. 2014;15(3):284-91.

33. Mitsuhashi T, Hibi K, Kosuge M, Morita S, Komura N, Kusama I, Otsuka F, Endo M, Iwahashi N, Okuda $\mathrm{J}$, et al. Relation between hyperinsulinemia and nonculprit plaque characteristics in nondiabetic patients with acute coronary syndromes. JACC Cardiovasc Imaging. 2011;4(4):392-401.

34. Virdis A, Santini F, Colucci R, Duranti E, Salvetti G, Rugani I, Segnani C, Anselmino M, Bernardini N, Blandizzi C, et al. Vascular generation of tumor necrosis factor-a reduces nitric oxide availability in small arteries from visceral fat of obese patients. J Am Coll Cardiol. 2011;58(3):238-47.

35. Plomgaard P, Bouzakri K, Krogh-Madsen R, Mittendorfer B, Zierath JR, Pedersen BK. Tumor necrosis factor-alpha induces skeletal muscle insulin resistance in healthy human subjects via inhibition of Akt substrate 160 phosphorylation. Diabetes. 2005;54(10):2939-45.

\section{Figures}


NSTE-ACS Patients underwent isolated OPCABG from 2019 Febrary to 2020 December in Beijng

Anzhen Hospital ( $\mathrm{n}=6315)$

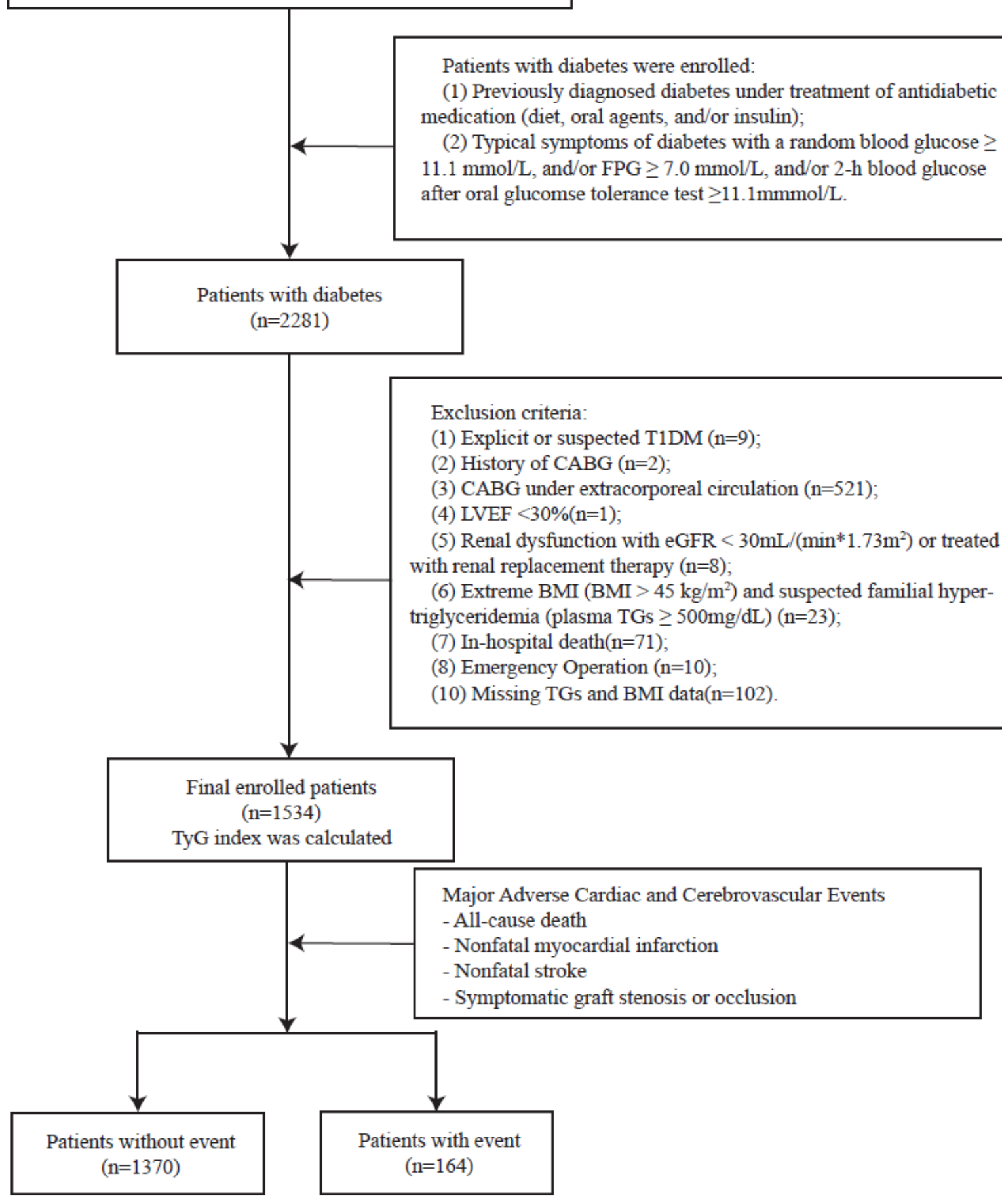

\section{Figure 1}

Flow chart of the study population enrollment. NSTE-ACS non-ST-segment elevation acute coronary syndrome, OPCABG off-pump coronary artery bypass grafting, FPG fasting plasma glucose, T1DM type 1 diabetes mellitus, CABG coronary artery bypass grafting, TGs triglycerides, eGFR estimated glomerular filtration rate, ALT alanine transaminase, AST aspartate transaminase, BMI body mass index, TyG triglyceride glucose 
a

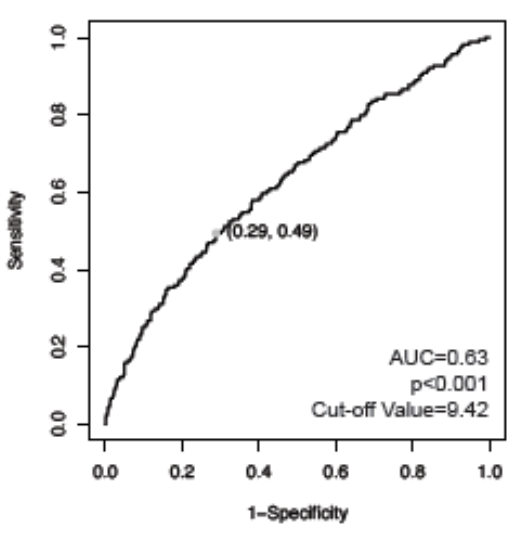

d

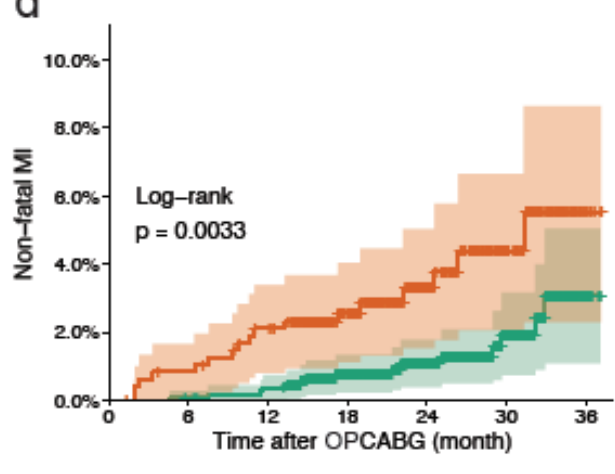

Num. at risk

$\begin{array}{ccccccc}\text { Lower } 1057 & 1055 & 1050 & 807 & 540 & 275 & 31 \\ \text { HIgher } 477 & 471 & 461 & 341 & 206 & 101 & 7\end{array}$

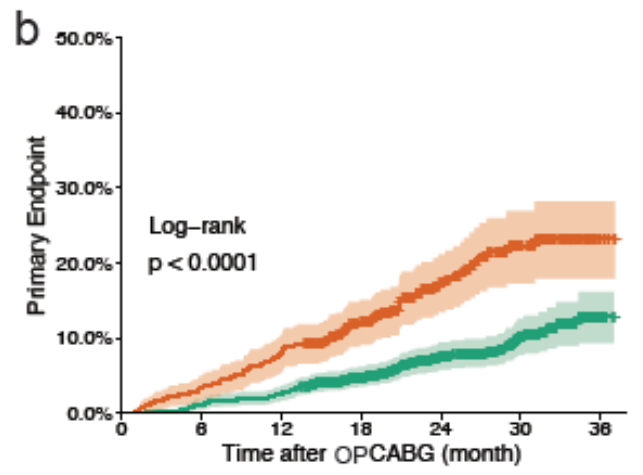

Num. at risk

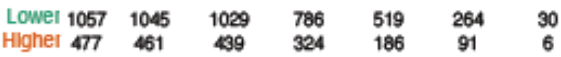

e

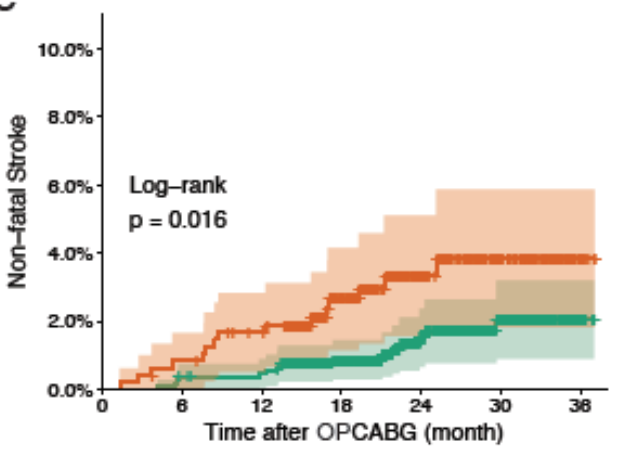

Num. at risk

$\begin{array}{lllllll}\text { Lower } 1057 & 1052 & 1049 & 806 & 537 & 272 & 30 \\ \text { Highel } 477 & 472 & 464 & 343 & 203 & 100 & 7\end{array}$

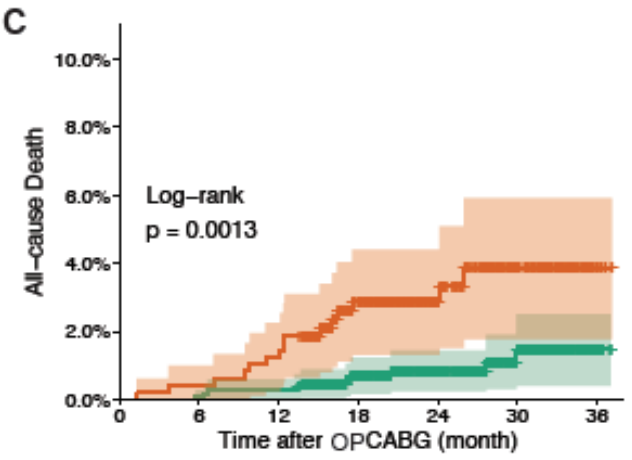

Num. at risk
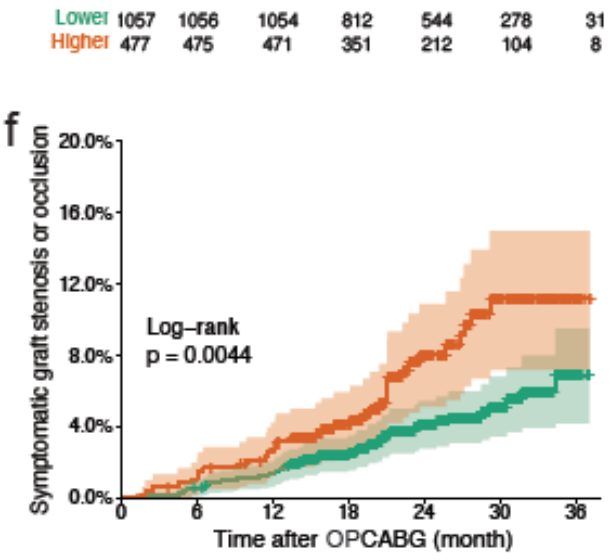

Num. at risk

$\begin{array}{lllllll}\text { Lower } 1067 & 1050 & 1040 & 797 & 590 & 271 & 31 \\ \text { Hohel } 477 & 470 & 458 & 343 & 202 & 98 & 8\end{array}$

Lower TyG index

Higher TyG index

\section{Figure 2}

Kaplan-Meier curves for endpoint events according to the optimal cutoff point of TyG index. a ROC curve for TyG index for primary endpoint; b Kaplan-Meier curves for primary endpoint; c Kaplan-Meier curves for all-cause death; d Kaplan-Meier curves for nonfatal Ml; e Kaplan-Meier curves for nonfatal stroke; $f$ Kaplan-Meier curves for symptomatic graft stenosis or occlusion. The groups were stratified by the optimal cutoff point of TyG index determined by ROC curve analysis. AUC Area Under Curve, TyG triglyceride glucose, OPCABG off-pump coronary artery bypass, MI myocardial infarction, HR hazard ratio, $\mathrm{Cl}$ confidence interval 
subgroup

\begin{tabular}{|c|c|}
\hline Age $\leq 65$ years & $921(60.0)$ \\
\hline Age $>65$ years & $613(40.0)$ \\
\hline male & $1092(71.2)$ \\
\hline female & $442(28.8)$ \\
\hline $\mathrm{BMI} \leq 28 \mathrm{~kg} / \mathrm{m}^{2}$ & $1166(76.0)$ \\
\hline $\mathrm{BMI}>28 \mathrm{~kg} / \mathrm{m}^{2}$ & $368(24.0)$ \\
\hline Hypertension- & $420(27.4)$ \\
\hline Hypertension+ & $1114(72.6)$ \\
\hline Diagnosis (UA) & $1451(94.6)$ \\
\hline Diagnosis (NSTEMI) & $83(5.4)$ \\
\hline $\mathrm{LDL}-\mathrm{C} \leq 70 \mathrm{mg} / \mathrm{dL}$ & $491(32.0)$ \\
\hline $\mathrm{LDL}-\mathrm{C}>70 \mathrm{mg} / \mathrm{dL}$ & $1043(68.0)$ \\
\hline Statin - & $134(8.7)$ \\
\hline Statin + & $1400(91.3)$ \\
\hline Oral hypoglycemic agents - & $905(59.0)$ \\
\hline Oral hypoglycemic agents + & $629(41.0)$ \\
\hline Insulin - & $1485(96.8)$ \\
\hline Insulin + & $49(3.2)$ \\
\hline LIMA - & $454(29.6)$ \\
\hline LIMA + & $1080(70.4)$ \\
\hline one or two bridges & $635(41.4)$ \\
\hline three or more bridges & $899(58.6)$ \\
\hline IABP - & 1507(98.2) \\
\hline $\mathrm{IABP}+$ & $27(1.8)$ \\
\hline
\end{tabular}

HR( $95 \% \mathrm{Cl})$

P for Interaction

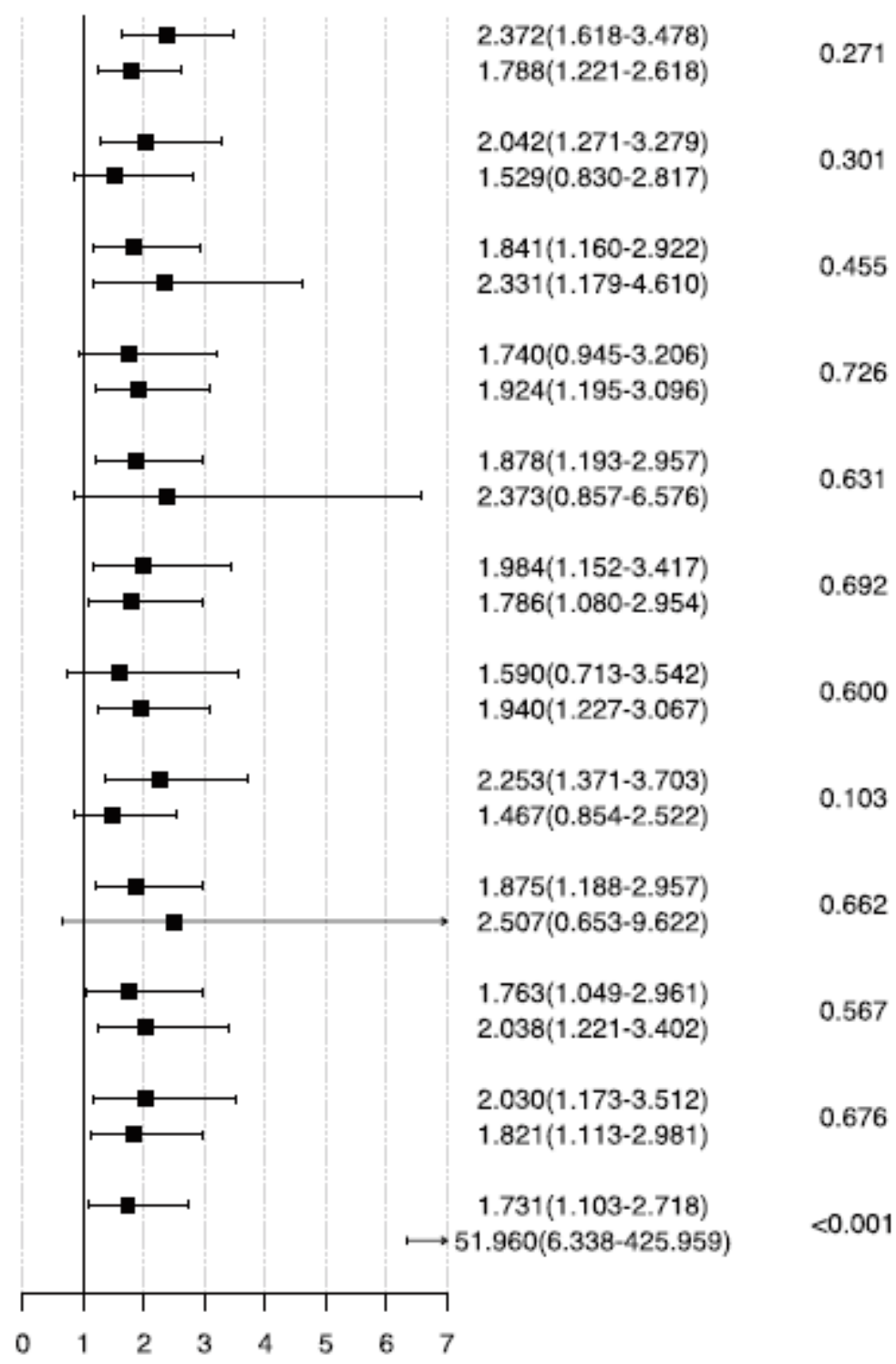

\section{Figure 3}

Cox proportional hazards analysis evaluating prognostic implication of TyG index in various stratifications. HR was evaluated by 1-un increase of TyG index. BMI body mass index, UA unstable angina, NSTEMI non-ST-segment elevation myocardial infarction, LDL-C low-density lipoprotein cholesterol, LIMA left internal mammary artery, IABP intra-aortic balloon pump, HR hazard ratio, $\mathrm{Cl}$ confidence interval

\section{Supplementary Files}

This is a list of supplementary files associated with this preprint. Click to download.

- TableS1.docx 